\title{
High-Pressure Phases of Nitrogen
}

\author{
Chris J. Pickard \\ Scottish Universities Physics Alliance, School of Physics and Astronomy, University of St. Andrews, \\ St. Andrews, KY16 9SS, United Kingdom \\ R. J. Needs \\ Theory of Condensed Matter Group, Cavendish Laboratory, Cambridge CB3 OHE, United Kingdom
} (Received 8 August 2008; revised manuscript received 21 December 2008; published 27 March 2009)

\begin{abstract}
Density-functional-theory calculations and a structure-searching method are used to identify candidate high-pressure phases of nitrogen. We find six structures which are calculated to be more stable than previously studied structures at some pressures. Our four new molecular structures give insight into the most efficient packings of nitrogen molecules at high pressures, and we predict two new nonmolecular structures to be stable at very high pressures.
\end{abstract}

DOI: 10.1103/PhysRevLett.102.125702

PACS numbers: 64.70.kt, 61.66.-f, 62.50.-p, 71.15.Nc

Phase diagrams of low atomic number elements have been widely studied, and the behavior of nitrogen has been of particular experimental [1-10] and theoretical [11-24] interest. At low pressures and temperatures, nitrogen forms van der Waals bonded molecular solids consisting of triplybonded $\mathrm{N}_{2}$ dimers with a very large dissociation energy of $9.76 \mathrm{eV}$, more than twice that of $\mathrm{H}_{2}$. Dissociation of $\mathrm{H}_{2}$ molecules has not been observed in high-pressure diamond-anvil-cell experiments. Nevertheless, pressureinduced dissociation of $\mathrm{N}_{2}$ molecules has been observed and nonmolecular nitrogen recovered to ambient conditions as a metastable high-energy density material (HEDM) [5,6].

Nitrogen atoms can form one, two, or three covalent bonds and make molecules, polymers, and dense framework structures, whereas a hydrogen atom can form only one covalent bond. This suggests that structures formed upon dissociating $\mathrm{N}_{2}$ at high pressures could be insulating, while those of hydrogen are likely to be metallic. In addition, the barriers to reforming $\mathrm{N}_{2}$ molecules from an insulating, covalently bonded nonmolecular structure are expected to be large, and metastable nonmolecular nitrogen can indeed be recovered, which is inconceivable in hydrogen. A HEDM consisting of energetic nonmolecular nitrogen could form a useful rocket propellant because the exhaust would be $\mathrm{N}_{2}$ molecules which are both environmentally safe and difficult to detect because of their similarity to the surrounding air [25].

Pressure-induced dissociation of $\mathrm{N}_{2}$ was suggested from density-functional-theory (DFT) calculations [11,12], and Mailhiot et al. [13] predicted that the nonmolecular "cubic gauche" structure would become stable at high pressures. A decade later, and after much experimental work [2-4,7], a transition from molecular to cubic gauche nitrogen was confirmed by Eremets et al. at pressures above $110 \mathrm{GPa}$ [5]. There is no experimental evidence for higher pressure phases, although theoretical studies have predicted the nonmolecular "black phosphorus" structure to be stable above $\sim 210 \mathrm{GPa}$ [13].

The phase diagram of nitrogen is complicated; gas and liquid phases, a number of molecular solid phases, and the high-pressure nonmolecular cubic gauche phase have been observed. A sequence of pressure-induced transitions between molecular phases occurs at low temperatures: $\alpha \stackrel{0.4 \mathrm{GPa}}{\longrightarrow} \gamma \stackrel{11 \mathrm{GPa}}{\longrightarrow} \epsilon \stackrel{18 \mathrm{GPa}}{\longrightarrow} \zeta[1]$. The low pressure and temperature $\alpha$ phase of $P a \overline{3}$ space-group symmetry consists of molecules whose centers lie on a face-centered-cubic (fcc) lattice. The molecular centers of the $\gamma$ phase form an approximately fcc lattice but with a tetragonal distortion, giving $\mathrm{P}_{2} / \mathrm{mnm}$ symmetry. The x-ray diffraction data for the $\epsilon$ phase are compatible with $R \overline{3} c$ symmetry, but the structure of the $\zeta$ phase is currently unknown $[1,8]$. Stable molecular phases have also been found at higher temperatures, most notably the $\beta, \delta$, and $\delta^{\prime}$ phases. $\zeta^{\prime}, \kappa, \eta, \theta$, and $\iota$ phases have also been reported [1,7,8], although their structures are unknown. Experimental studies of nitrogen structures at high pressures are challenging because of its relatively weak x-ray scattering, and calculations can help to determine the favorable types of molecular packing.

One of the major discrepancies between experiment and theory is that molecular nitrogen has been studied in diamond anvil cells at pressures above $100 \mathrm{GPa}$, whereas DFT calculations indicate that nonmolecular cubic gauche is more stable above $\sim 50 \mathrm{GPa}$. The resolution of this discrepancy might lie in the use of an approximate DFT, the use of incorrect $\mathrm{N}_{2}$ structures in the calculations, or the existence of large kinetic barriers to the dissociation of $\mathrm{N}_{2}$ molecules and to their reformation from nonmolecular material. Cubic gauche nitrogen has been synthesized by laser heating at pressures of 110-130 GPa $[5,9,10]$, which sets an upper limit on the molecular or cubic gauche equilibrium pressure. However, nonmolecular material has been obtained on releasing pressure from cubic gauche $[5,6]$, and a lower limit on the equilibrium pressure has not 
been determined. As the structure of the high-pressure $\zeta$ molecular phase is currently unknown, DFT calculations have used less than optimal structures, and will have underestimated the equilibrium pressure. Gregoryanz et al. suggested that using the true molecular structure in DFT calculations would shift the equilibrium pressure above $100 \mathrm{GPa}$ [8].

We have performed DFT calculations to help clarify the high-pressure phase diagram of nitrogen. Our searches involve relaxing ensembles of randomly chosen structures to minima in the enthalpy at fixed pressure [26-28]. Recent successes of our approach include the prediction of a highpressure insulating phase of silane [26] which was later observed in experiments [29] and our prediction of a metallic phase of aluminum hydride [28] which was subsequently observed [30]. The initial cells are generated by choosing random unit cell translation vectors and renormalizing their volumes to lie within a reasonable range. In the first stage of the search, we place the atoms at random positions within each cell and relax the cell shape and atomic positions. In the second stage, we apply constraints to bias the search towards regions likely to contain the most stable structures. We employ constraints of two types. In the first type, we place $\mathrm{N}_{2}$ molecules randomly within the cells, rejecting structures in which the molecules overlap strongly, and then relax. In the second type, we place atoms or molecules randomly within the cell and generate the full structure using a particular space group symmetry. We reject structures with strongly overlapping molecules and relax while maintaining the space group symmetry (the structures can relax into higher-symmetry space groups).

We used the CASTEP plane-wave code [31], ultrasoft pseudopotentials [32], and the Perdew-Burke-Ernzerhof (PBE) Generalized Gradient Approximation (GGA) density functional [33]. For the searches, we used a planewave basis set cutoff of $250 \mathrm{eV}$ and performed the Brillouin zone integrations using a $k$-point grid of spacing $2 \pi \times$ $0.07 \AA^{-1}$. The most interesting structures were further relaxed at a higher level of accuracy consisting of a basis set cutoff of $500 \mathrm{eV}$, a k-point grid of spacing $2 \pi \times$ $0.03 \AA^{-1}$, and a somewhat harder pseudopotential which included nonlinear core corrections.

We performed unconstrained searches at 50, 75, 100, 200, and $300 \mathrm{GPa}$, with 4, 6, 8, and 12 atoms per cell (not all combinations of these pressures and numbers of atoms were studied), using up to 1000 initial structures per system. To study the molecular regime in more detail, we performed extensive searches at $25 \mathrm{GPa}$. We used initial structures of 6 or 8 atoms per cell which were constrained to form $\mathrm{N}_{2}$ molecules. Further searches were performed at $25 \mathrm{GPa}$ in 12 -atom cells constrained to the $P 2_{1}$ space group which contains two operations and in 8-atom cells constrained to the $P 2_{1}$ space group and to the $P \overline{1}$ space group (two operations). These searches generated the observed cubic gauche nonmolecular phase (space group $I 2{ }_{1} 3$ ) and the "black phosphorus" ( $\mathrm{Cmca}$ ) structure. We also found new low-enthalpy nonmolecular structures of space groups $P \overline{4} 2_{1} m$ and $P 2_{1} 2_{1} 2_{1}$, and numerous molecular structures, including new low-enthalpy ones of $P b c n, P 2_{1} / c, C 2 / c$, and $P 4_{1} 2{ }_{1} 2$ symmetries.

The enthalpy-pressure relations of the more stable phases, calculated at the higher level of accuracy, are plotted in Fig. 1, and the details of the structures are given in the auxiliary material [34]. In the pressure range $0-0.23 \mathrm{GPa}$, the $\operatorname{Pa} \overline{3}(\alpha)$ phase is the most stable, in agreement with experiment. The $P 4_{2} / \mathrm{mnm}(\gamma)$ molecular phase is very competitive at low pressures, and becomes more stable than $P a \overline{3}$ at $0.47 \mathrm{GPa}$, in good agreement with experiment, although at this pressure the $P b c n$ and $P 2_{1} / c$ molecular phases are $0.6 \mathrm{meV}$ per atom lower in enthalpy. At low pressures, $P b c n$ and $P 2_{1} / c$ are more stable than $C 2 / c$ and $P 4_{1} 2{ }_{1} 2$, while at higher pressures, all four struc-
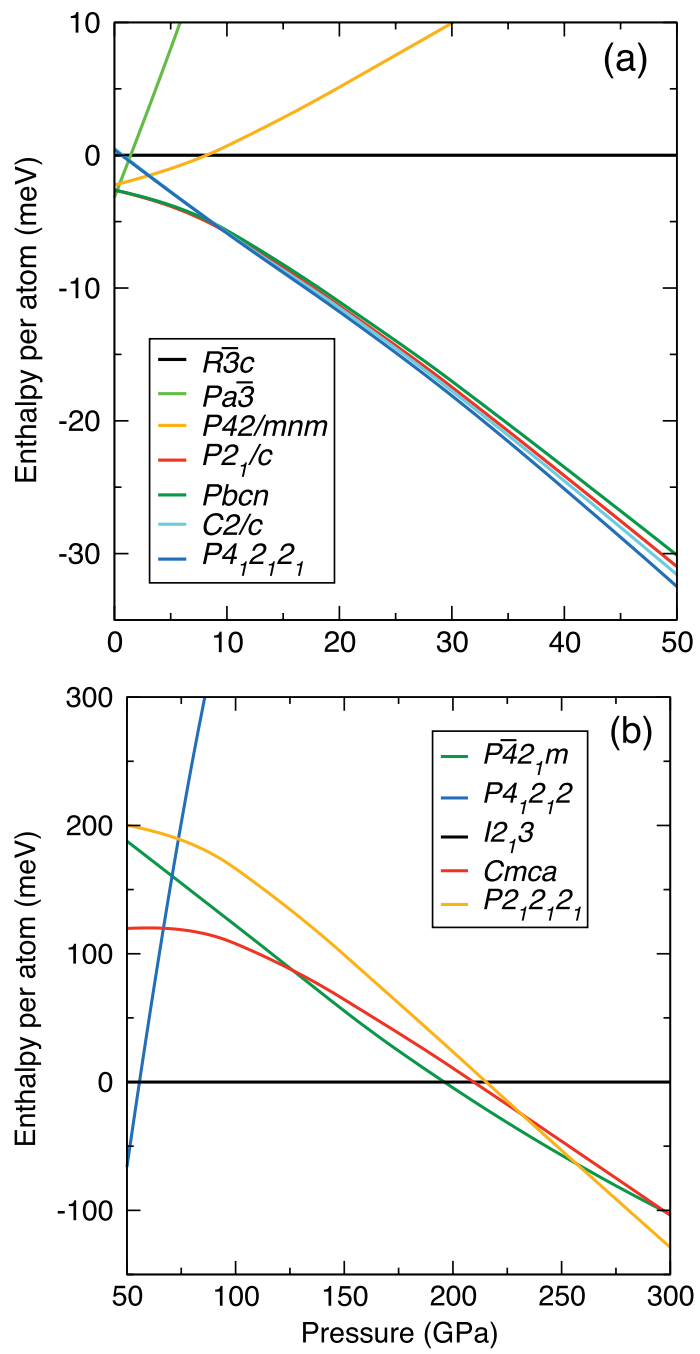

FIG. 1 (color). Variation of the enthalpies of various phases of nitrogen with pressure. Differences in enthalpy from the $R \overline{3} c$ phase are plotted in the pressure range 0-50 GPa (a), and from the cubic gauche $I 2{ }_{1} 3$ phase in the range 50-300 GPa (b). 
tures are nearly degenerate, although $P 4_{1} 2_{1} 2$ becomes the most stable phase above $\sim 9.5 \mathrm{GPa}$. The $P 4_{1} 2{ }_{1} 2 /$ cubic gauche equilibrium pressure is calculated to be $56 \mathrm{GPa}$. The $R \overline{3} c$ structure, which has been identified with the $\epsilon$ phase, is never stable in our calculations, although within the low-temperature stability range of the $\epsilon$ phase (11$18 \mathrm{GPa}$ ), it is only 6-11 meV per atom higher in enthalpy than our most stable phase $\left(P 4_{1} 2_{1} 2\right)$.

The $P 2_{1} / c$ and $P 4_{1} 2_{1} 2$ structures are illustrated in Fig. 2, while $P b c n$ and $C 2 / c$ are pictured in the auxiliary material [34]. The molecular centers of $P b c n$ form an approximately hcp lattice with small displacements from the ideal positions and a small cell distortion. The molecular centers of $P 4_{1} 2_{1} 2$ form an approximately fcc lattice, although the displacements from the ideal positions are larger than in Pbcn. C2/c is also based on an fcc lattice, but only half the molecules are displaced, and the accompanying cell distortion is significant. $P 2_{1} / c$ is also based on an fcc lattice, but with molecular displacements and a significant cell distortion.

The relative stabilities of the compressed molecular structures are related to their packing. The packing efficiency can be measured by $P=d^{3} / V$, where $d$ is the shortest distance between atoms on neighboring molecules and $V$ is the volume per molecule. At $50 \mathrm{GPa}$, we find $P=$ 0.677 for the $R \overline{3} \mathrm{c}$ and $P 4_{2} / \mathrm{mnm}$ structures, $P=0.715$ for $C 2 / c$ and $P 4_{1} 22_{1} 2, P=0.719$ for $P b c n$, and $P=0.722$ for

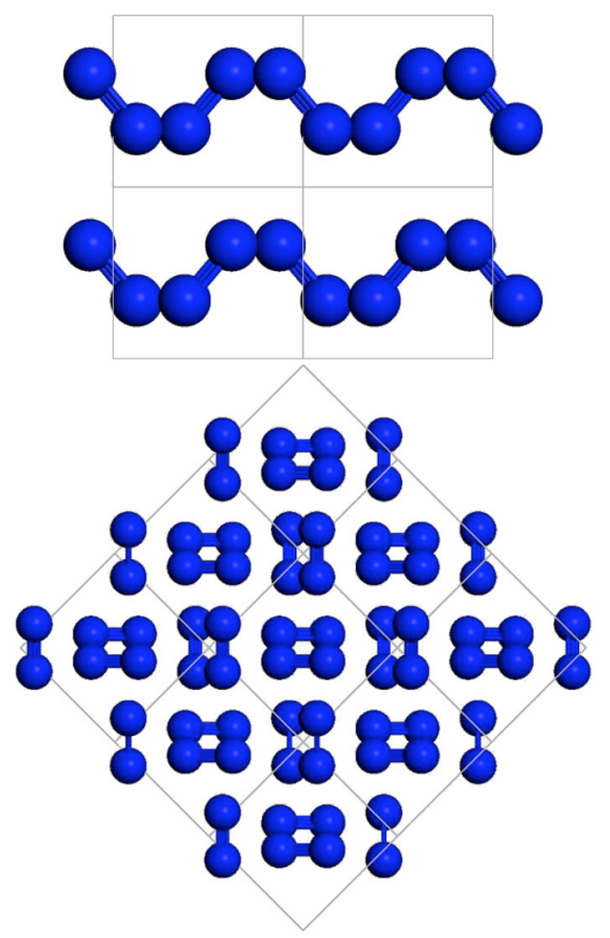

FIG. 2 (color online). The molecular $P 2_{1} / c$ phase (top) and the molecular $P 4_{1} 2{ }_{1} 2$ phase (bottom) at $40 \mathrm{GPa}$. A fuller appreciation can be obtained by viewing the structures given in the auxiliary material [34] with a visualization package.
$P 2_{1} / c$. The parameter $P$ distinguishes between the $R \overline{3} c$ and $P 4_{2} / \mathrm{mnm}$ structures and the very-well-packed molecular structures we have found.

Cubic gauche is calculated to be stable in the range 56$196 \mathrm{GPa}, P \overline{4} 2_{1} m$ in the range $196-257 \mathrm{GPa}$, and $P 2_{1} 2_{1} 2_{1}$ becomes the most stable above $257 \mathrm{GPa}$. We find no region of stability for the Cmca "black phosphorus" structure; Fig. 1(b) shows that it is unstable below $300 \mathrm{GPa}$, and it is not expected to be stable at higher pressures because it is less dense than $P 2_{1} 2_{1} 2_{1}$. (The inverses of the slopes of the curves in Fig. 1 give the density differences from the reference phases.) Cubic gauche is a high-symmetry three-dimensional threefold-coordinated framework structure in which all atoms are equivalent [13]. $P \overline{4} 2_{1} m$ is a layered structure with two inequivalent atomic sites and threefold coordination, as pictured in Fig. 3. $P 2_{1} 2_{1} 2_{1}$ is a three-dimensional framework structure with two inequivalent atomic sites and threefold-coordinated atoms forming square spirals which are bonded to each other, see Fig. 3.

At $300 \mathrm{GPa}$, our new high-pressure phases have band gaps of $3.5 \mathrm{eV}\left(P \overline{4} 2_{1} m\right)$ and $3.0 \mathrm{eV}\left(P 2_{1} 2_{1} 2_{1}\right)$, which are substantially larger than the band gap of $0.9 \mathrm{eV}$ of "black phosphorus" nitrogen, which was previously suggested to be stable. This suggests that, even at $300 \mathrm{GPa}$, nitrogen is very far from becoming metallic at low temperatures.

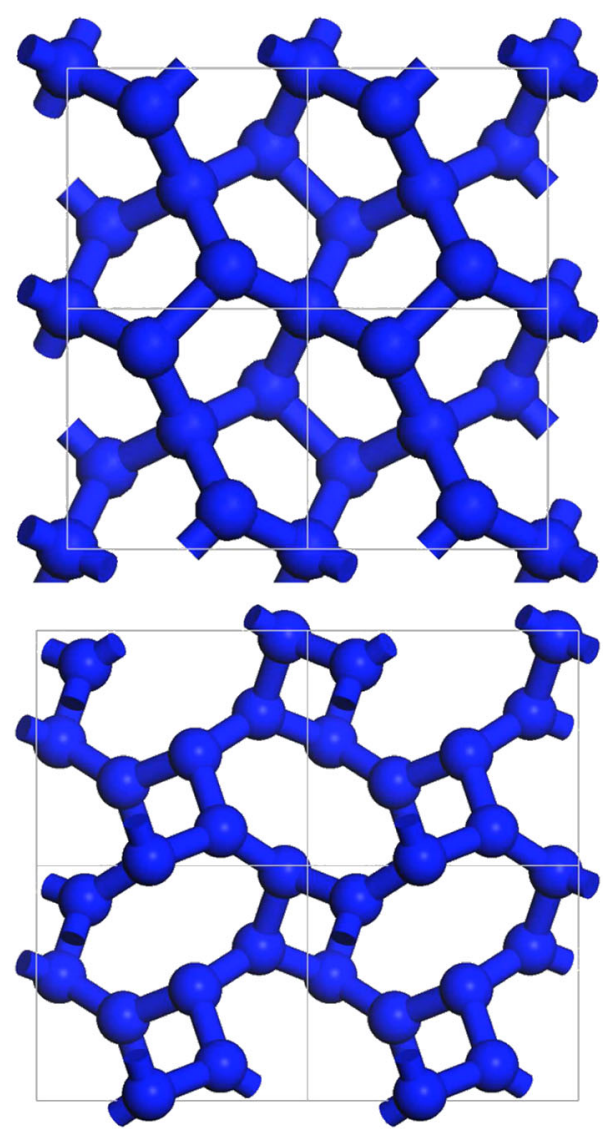

FIG. 3 (color online). The nonmolecular $P \overline{4} 2_{1} m$ (top) and $P 2{ }_{1} 2_{1} 2_{1}$ structures (bottom) at $200 \mathrm{GPa}$. 
Some phases were recalculated using the local density approximation (LDA) to test sensitivity to the density functional. The relative enthalpies of the molecular phases are not much changed by using the LDA and, for example, at $50 \mathrm{GPa}$, the enthalpy difference between $P 2_{1} / c$ and $P 4_{1} 2_{1} 2$ is increased by about $1 \mathrm{meV}$ per atom. As our calculated low-pressure phase diagram is in fair agreement with experiment, we conclude that the errors due to the approximate density functional cancel strongly between the molecular phases. The enthalpy differences between some molecular phases are very small, and effects such as van der Waals interactions which are not described by the PBE and LDA functionals may be significant at pressures of a few GPa. van der Waals interactions are not expected to be important in determining the structures adopted at higher pressures because they vary slowly with interatomic distance compared with the massive repulsive forces. The energy scale of these repulsive forces is given by the $p V$ term in the enthalpy, which is already $2.5 \mathrm{eV}$ per atom at $50 \mathrm{GPa}$.

The enthalpy differences between the molecular and nonmolecular phases are more sensitive to the density functional, and using the LDA instead of the PBE gives a $P 4{ }_{1} 2{ }_{1} 2$ /cubic gauche equilibrium pressure of $33 \mathrm{GPa}$, which corresponds to an upwards shift of the $P 4_{1} 2_{1} 2$ phase on Fig. 1(b) of about $280 \mathrm{meV}$ per atom. To obtain an equilibrium pressure of $100 \mathrm{GPa}$ would require a downwards shift of the enthalpy of $P 4_{1} 2_{1} 2$ by $410 \mathrm{meV}$ per atom on Fig. 1(b). Calculations using other density functionals [17] and quantum Monte Carlo methods [14] do not support such a large shift. At $56 \mathrm{GPa}$, our $P 4_{1} 2_{1} 2$ molecular structure is $36 \mathrm{meV}$ per atom lower in enthalpy than the $R \overline{3} c$ structure, which amounts to an increase in the molecular or cubic gauche equilibrium pressure of only about $3 \mathrm{GPa}$. We find it implausible that another molecular phase could exist with an enthalpy sufficiently lower than $P 4_{1} 2_{1} 2$ to increase the equilibrium pressure to $100 \mathrm{GPa}$. We conclude that the large difference between the theoretical equilibrium pressure and the experimental dissociation pressures arises from the large kinetic barriers between molecular and nonmolecular phases.

The LDA and PBE results for the nonmolecular phases are in reasonable agreement, the enthalpy differences at $200 \mathrm{GPa}$ from using the LDA amount to downwards shifts of $35 \mathrm{meV}$ per atom for $P \overline{4} 2_{1} m$ and $43 \mathrm{meV}$ for $P 2_{1} 2_{1} 2_{1}$ on Fig. 1(b). We conclude that using LDA results instead of PBE ones would not change our conclusions significantly.

Numerous DFT studies of compressed nitrogen have been reported, including several recent contributions aimed at predicting high-pressure phases [15,16,18-24]. However, since the ground-breaking DFT study of Mailhiot et al. in 1992 [13], no new stable structures have been predicted. Our prediction of six new lowenthalpy structures represents a significant step forward in understanding the phase diagram of nitrogen and in the prediction of structures using first-principles calculations.

The authors were supported by the Engineering and Physical Sciences Research Council (EPSRC) of the UK.

[1] R. Bini et al., J. Chem. Phys. 112, 8522 (2000).

[2] A. F. Goncharov et al., Phys. Rev. Lett. 85, 1262 (2000).

[3] M. I. Eremets et al., Nature (London) 411, 170 (2001).

[4] E. Gregoryanz et al., Phys. Rev. B 64, 052103 (2001).

[5] M. I. Eremets et al., Nature Mater. 3, 558 (2004).

[6] M. I. Eremets et al., J. Chem. Phys. 121, 11296 (2004).

[7] E. Gregoryanz et al., Phys. Rev. B 66, 224108 (2002).

[8] E. Gregoryanz et al., J. Chem. Phys. 126, 184505 (2007).

[9] M. I. Eremets, A. G. Gavriliuk, and I. A. Trojan, Appl. Phys. Lett. 90, 171904 (2007).

[10] M. J. Lipp et al., Phys. Rev. B 76, 014113 (2007).

[11] A. K. McMahan and R. LeSar, Phys. Rev. Lett. 54, 1929 (1985).

[12] R. M. Martin and R. J. Needs, Phys. Rev. B 34, 5082 (1986).

[13] C. Mailhiot, L.H. Yang, and A.K. McMahan, Phys. Rev. B 46, 14419 (1992).

[14] L. Mitas and R. M. Martin, Phys. Rev. Lett. 72, 2438 (1994).

[15] W. D. Mattson et al., Phys. Rev. Lett. 93, 125501 (2004).

[16] F. Zahariev et al., Phys. Rev. B 72, 214108 (2005).

[17] J. Uddin, V. Barone, and G. E. Scuseria, Mol. Phys. 104, 745 (2006).

[18] F. Zahariev et al., AIP Conf. Proc. 845, 373 (2006).

[19] R. Caracas and R. J. Hemley, Chem. Phys. Lett. 442, 65 (2007).

[20] F. Zahariev et al., Phys. Rev. Lett. 97, 155503 (2006).

[21] A. R. Oganov and C.W. Glass, J. Chem. Phys. 124, 244704 (2006).

[22] F. Zahariev et al., Phys. Rev. B 75, 140101(R) (2007).

[23] X. L. Wang et al., J. Phys. Condens. Matter 19, 425226 (2007).

[24] Y. Yao, J. S. Tse, and K. Tanaka, Phys. Rev. B 77, 052103 (2008).

[25] M. B. Talawar et al., Combust. Explos. Shock Waves 41, 264 (2005).

[26] C. J. Pickard and R. J. Needs, Phys. Rev. Lett. 97, 045504 (2006).

[27] C. J. Pickard and R. J. Needs, Nature Phys. 3, 473 (2007).

[28] C. J. Pickard and R. J. Needs, Phys. Rev. B 76, 144114 (2007).

[29] M. I. Eremets et al., Science 319, 1506 (2008).

[30] I. Goncharenko et al., Phys. Rev. Lett. 100, 045504 (2008).

[31] S. J. Clark et al., Z. Kristallogr. 220, 567 (2005).

[32] D. Vanderbilt, Phys. Rev. B 41, 7892 (1990).

[33] J. P. Perdew, K. Burke, and M. Ernzerhof, Phys. Rev. Lett. 77, 3865 (1996).

[34] See EPAPS Document No. E-PRLTAO-102-004915 for further information on the transition pressures and structures. For more information on EPAPS, see http://www. aip.org/pubservs/epaps.html. 